\title{
Caracterización de hatos ganaderos en la cuenca baja del río Shanusi Alto Amazonas - Loreto - Perú
}

Characterization of livestock herds in the lower basin of the Shanusi river Alto Amazonas - Loreto - Peru

Marco Mathios Flores ${ }^{1}$, Julio César Alegre Orihuela² y José Aguilar Vásquez ${ }^{1}$

\section{RESUMEN}

El objetivo fue la caracterización de los hatos ganaderos productores de leche de la cuenca baja del rio Shanusi Alto Amazonas - Loreto-Perú. En la metodología se aplicó encuestas a 26 ganaderos, se usó la estadística descriptiva, para la tipificación se aplicó el análisis de conglomerado de K-medias. Las variables cuantitativas: producción de leche 6,33 litros/vaca, el precio S/ 0,86/litro teniendo en producción 5 vacas/ hato, promedio de 14 has con pasturas mejoradas, la carga animal de 1,8 UA/ha, la mano de obra en promedio de 0,08 obreros. Respecto a las variables cualitativas: el $69,2 \%$ de pariciones fueron de mayo-octubre el 96,2\%, realiza un solo ordeño, $65,4 \%$ maneja registro, el 100\% no realiza control de calidad, y el 53,8\% cuenta con sala de ordeño. Social-Educativo, el 50\% de los propietarios realiza otras actividades agrícolas, 42,3\% de los propietarios tienen educación primaria y los obreros el 65,4\%. La especie forrajera, la Brachairia brizantha con 80,8 \% y el 100\% suministra sal mineral. Se Tipificaron tres conglomerados de crianzas extensivas. El G1 media producción con 46,15\%, el G2 baja producción con el 42,3\% y G3 mayor producción el 11,53\%. El nivel educativo de los propietarios dificulta la adopción de tecnologías.

Palabras claves: sistemas de producción; vacas; producción de leche; conglomerados

1 Universidad Nacional Autónoma de Alto Amazonas. Amazonas, Perú.

2 Universidad Nacional Agraria La Molina. Lima, Perú.

(C) Los autores. Este artículo es publicado por la Revista Aporte Santiaguino de la Universidad Nacional Santiago Antúnez de Mayolo. Este es un artículo de acceso abierto, distribuido bajo los términos de la Licencia Creative Commons Atribución-NoComercial-CompartirIgual 4.0 Internacional. (http://creativecommons.org/licenses/ by-nc-sa/4.0/), que permite el uso no comercial, distribución y reproducción en cualquier medio, siempre que la obra original sea debidamente citada. 


\begin{abstract}
The objective was the characterization of the milk producing cattle herds of the lower basin of the river Shanusi Alto Amazonas - Loreto - Peru. In the methodology, surveys were applied to 26 breeders, the descriptive statistics was used, for the typification, the K-means cluster analysis was applied. The quantitative variables: milk production 6,33 liters/cow, the price S/ 0,86/liter having in production 5 cows/herd, average of 14 has with improved pastures, the animal load of 1,8 AU / ha , the average labor force of 0,08 workers. Regarding the qualitative variables: $69,2 \%$ of births were from May to October, 96,2\%, performed only one milking, 65,4\% handled registration, $100 \%$ did not perform quality control, and 53,8\% did has a milking parlor. Social - Educational, $50 \%$ of the owners carry out other agricultural activities, $42,3 \%$ of the owners have primary education and the workers 65,4\%. The forage species, Brachiaria brizantha with $80,8 \%$ and $100 \%$ supplies mineral salt. Three conglomerates of extensive breeding were typified. The G1 media production with 46,15\%, the G2 low production with $42,3 \%$ and G3 higher production 11,53\%. The educational level of the owners makes it difficult to adopt technologies.
\end{abstract}

Keywords: production systems; cows; milk production; conglomerates

\title{
INTRODUCCIÓN
}

Los parámetros de producción de leche son definitivamente alterados por las condiciones ambientales presentes y cambiantes en las diferentes condiciones agroecológicas y de manejo de las fincas ganaderas afirman, Salamanca y Bentez (2012); igualmente, la genética de los animales modifica los índices de producción, indicando que la interacción entre el genotipo y el ambiente es un factor que debe tenerse en cuenta para la implementación de sistemas productivos. Existe relación entre variables cualitativas y cuantitativas, dependiendo si el productor es considerado pequeño, mediano o grande según los estudios de Avilez et al. (2010). La cuenca baja del río Shanusi es un sector de mucha importancia económica de la provincia de Alto amazonas por su producción agropecuaria, sin embargo, actualmente las actividades están siendo muy cambiantes a cultivos agroindustriales, al parecer se está soslayando la actividad ganadera. Se justifica la investigación porque es necesario que el productor conozca qué características cualitativas y cuantitativas productivas que tiene su hato ganadero productor de leche para que tome decisiones pertinentes en la mejora de su producción y del ambiente. El objetivo general del presente trabajo de investigación fue, Caracterizar los hatos ganaderos productores de leche de la cuenca baja del río Shanusi Alto Amazonas - Loreto - Perú. 


\section{MATERIALES Y MÉTODOS}

\section{Lugar de ejecución del trabajo}

La investigación se desarrolló en la cuenca baja del río Shanusi ubicado en el distrito de Yurimaguas, provincia de Alto Amazonas, región Loreto ubicado a una altitud de 181.51 m.s.n.m. Es una zona tropical húmeda con altas temperaturas con un promedio anual de $26,3^{\circ} \mathrm{C}$, con un promedio anual de lluvias entre los 3000 a $4000 \mathrm{~mm}$ anuales. Geográficamente se localiza entre las coordenadas $05^{\circ} 53^{\prime} 34^{\prime \prime}$ de Latitud Sur y $76^{\circ} 06^{\prime}$ 36" de Longitud Oeste.

\section{Metodología}

Esta investigación es de tipo descriptivo, exploratorio y explicativo que permitió caracterizar los hatos ganaderos productores de leche de la cuenca baja del rio Shanusi Alto Amazonas - Loreto - Perú. Para determinar la población y muestra en el trabajo de investigación se tuvo en cuenta a 26 hatos ganaderos miembros de la Asociación de Ganaderos Lecheros de Yurimaguas ubicados en la cuenca baja del rio Shanusi. Se seleccionó hatos lecheros que cuenten con más de diez animales. La toma de datos se realizó mediante una entrevista in situ a los propietarios de los hatos ganaderos utilizando una encuesta a través de una ficha que permitió registrar los datos, para evaluar las variables cuantitativas y cualitativas. El procesamiento de datos se realizó mediante la estadística descriptiva (promedios, frecuencias, porcentajes, etc.) y el análisis de conglomerados (AC) de K-medias para tipificar a los grupos de explotaciones lecheras similares entre sí, en términos de las variables cuantitativas empleadas para clasificarlas.

Variables cuantitativas:

- Edad del producto (Años): Corresponde a los años dedicados a crianza de ganado vacuno.

- Producción de leche/vaca/día. Corresponde a la producción diaria de leche por vaca.

- Número de vacas en producción en hatos ganadero $(\mathrm{V} / \mathrm{h})$ : Número de vacas en producción de leche en hatos ganaderos.

- Área pasturas mejoradas/pasturas totales. Razón entre las superficies mejoradas y la superficie total de pasturas. con un tiempo de producción igual o menor a cinco años.

- Unidades animales (UA): (vacas lecheras + toros + vaquillas y terneras de reemplazo).

- Carga animal. Se calculó dividiendo el tamaño del hato lechero (vacas lecheras + toros + vaquillas y terneras de reemplazo) por la superficie destinada al rubro (pasturas).

- Mano de obra. El número de trabajadores empleados anualmente en el rubro lechero por cada explotación. Los trabajadores temporales fueron agregados en una proporción equivalente al número de meses que trabajaron en la explotación. 
- Fertilización (Kg/ha). Kilogramos de fertilizantes por hectárea.

- Porcentaje de ganado de doble propósito (\%) cruces europeos x cebuinos.

Variables cualitativas:

\section{Manejo}

- Época de pariciones. Clasificada en dos categorías: mayor precipitación (noviembre-abril) y menor precipitación (mayo- octubre).

- Número de ordeño. Indica el número de ordeños por día que realiza el productor.

- Registros de producción.

- Control de calidad. Es el reporte del análisis bromatológico y microbiológico.

- Equipo e instalaciones.

Social - Educativo

- Dedicación a la actividad lechera del productor. Tres categorías: Exclusiva, Parcial + otras actividades agropecuarias, y Parcial + actividades no agropecuarias.

- Nivel de estudios del propietario. Cinco categorías: Sin estudios, Primaria (completa o incompleta), secundaria (completa o incompleta), técnica superior (completa o incompleta), superior universitario (completa o incompleta).

- Grado de instrucción de los obreros. Cuatro categorías: Sin estudios, Primaria, secundaria, técnico productivo.

Alimentación

- Pasturas mejoradas de pastoreo: pasturas introducidas.

- Pasto de corte. Complementación de forraje.

- Suplementación mineral. Inclusión de sal mineral en la dieta.

- Alimento balanceado. Se incluye para potenciar la producción de leche.

\section{RESULTADOS Y DISCUSIONES}

\section{Características cuantitativas de los hatos}

En la tabla 1 se muestra que, la edad promedio de los productores es 51 años, encontrándose en la última fase de su mayor rendimiento físico para la agricultura, especialmente en la ganadería en términos generales, sin embargo, cuentan con mayor experiencia, similar a lo encontrado por Andrade et al. (2008). La producción de leche en promedio fue 6 litros/vaca/día, la cual es un rendimiento bajo y que está influenciado por varios factores, como alimentación, salud, manejo y calidad genética y fue bastante mayor a lo reportado en la Amazonía ecuatoriana con una producción promedio de leche de 3,3 litros/vaca/día (Vera y Riera, 2004); por el contrario Vásquez (2007) en un trabajo de caracterización realizado en el eje carretero Yurimaguas Munichis reportó 
una producción promedio de 10 litros/vaca/día, y que es superior a este trabajo, esto debido principalmente a la calidad genética con que trabajan los ganaderos siendo en su mayoría el cruce de las razas Holstein con Gyr lechero. El precio de comercialización de la leche en promedio es de S/ 0,86, los productores refieren que el precio en el lugar de ordeño es de S/ 0,80 y en la ciudad es de S/ 1,2; la infraestructura de conectividad de los predios a los lugares de comercialización es deficiente, además no existe centros de acopios, actualmente los programas sociales han dejado de comercializar leche fresca, siendo remplazado por otros productos, por lo que no existe mercados fijos. Requelme y Bonifaz (2012), indican que existe un precio fijado por el Estado de $\$$ 0,39 en Ecuador, que es superior a lo que se encontró. En Tarapoto - Perú se comercializa a S / 0,80 a la empresa privada y entre S/ 1,00 a S/ 1,50 a los Programas Sociales (Vaso de Leche y PRONAA) Ruiz, (2011).

Tabla 1. Promedio, desviación estándar e intervalo de confianza 95\% para las variables cuantitativas consideradas en el censo $(n=26)$ para la caracterización de hatos lecheros

\begin{tabular}{lrrrr}
\hline \multicolumn{1}{c}{ VARIABLES } & PROMEDIO & DS & MÍNIMO & MÁXIMO \\
\hline Edad del productor & 50,83 & 11,0 & 27,0 & 70,0 \\
Producción de leche & 6,33 & 4,5 & 0,0 & 19,0 \\
Precio de la leche & 0,86 & 0,1 & 0,8 & 1,2 \\
Vaca en producción & 5,04 & 4,1 & 0,0 & 13,0 \\
Pasturas mejoradas & 14,50 & 16,0 & 0,0 & 80,0 \\
Fertilización & 10,8 & 37,6 & 0,0 & 150 \\
Pasturas no & 5,75 & 6,5 & 0,0 & 27,0 \\
mejoradas & 37,23 & 45.2 & 11,8 & 228,0 \\
Unidad animal & 1,80 & 0,7 & 0,8 & 3,2 \\
Carga animal & 17,25 & 8,9 & 5,0 & 43,0 \\
Áreas de pasturas & 16.25 & 11,4 & 8,0 & 60,0 \\
Vacas & 1,17 & 0,5 & 0,0 & 2,0 \\
Toros & 4,33 & 3,4 & 0,0 & 12,0 \\
Toretes & 6,63 & 7,2 & 0,0 & 35,0 \\
Vaquillas & 6,38 & 4,7 & 0,0 & 20,0 \\
Terneros & 34,88 & 22,7 & 14,0 & 120,0 \\
Total vacunos & 42,25 & 22,1 & 0,0 & 80,0 \\
Vacuno europeos & 53,17 & 22,4 & 60,0 & 100,0 \\
Vacuno cebuíno & 0,08 & 0,0 & 0,0 & 0,2 \\
Obreros & & & \\
\hline
\end{tabular}


Se encontró en promedio 5 vacas en producción, en la mayoría de los hatos los productores están reduciendo el número vacas probablemente debido a las pocas oportunidades de comercializar y a los altos costos de producción. Este número de vacas es por debajo de lo encontrado por Herrera (2009). Aproximadamente hace tres décadas en la provincia de Alto Amazonas se promovió el establecimiento de especies forrajeras mejoradas para pastoreo en la que se introdujo la Brachiaria decumbens, B. bumidicola, $B$. dictyoneura, la que tuvo mayor difusión fue la $B$. decumbens y actualmente la $B$. brizantha como forraje prometedor que ayuda sobre todo a la producción de leche: el promedio de pasturas mejoradas es de 14,5 ha; en algunos casos no tienen pasturas mejoradas y en otros llegar a tener hasta 80 ha; sin embargo en el Departamento de Meta Colombia los pequeños productores de escasa infraestructura cuentan con mayor promedio de pasturas mejoradas (Herrera, 2009). Respecto a la fertilización no es significativo, solo dos productores realizan una aplicación de 150 de NPK/ha, sin ningún análisis de suelo ni foliar, por lo que el forraje muestra deficiencia nutritiva dando como resultado baja calidad para una buena producción lechera.

En promedio los hatos ganaderos cuentan con 37 UA con un mínimo de 11,8 y un máximo de 228 y la carga animal es de 1,8 UA/ha, son productores pequeños, por lo que no producen mayor volumen de leche, para satisfacer mercados mayores, refieren que no realizan rotación de potreros, dejando de aprovechar pastura óptimamente, el problema mayor se da en épocas de menor precipitación pluvial por la poca producción foliar. Ríos (2007) indica que con un buen manejo se puede lograr 3 - 4 UA/ha/ año según los paquetes tecnológicos propuestos en Tarapoto y Pucallpa. El aspecto ambiental, como el genético son determinantes en la producción de leche, en este caso el cruce de vacunos europeos en promedio 42,25 y cebuinos 53,17 respectivamente, vacas cruzadas Bos taurus $\mathrm{x}$ Bos indicus de un grado intermedio de herencia europea son recomendables para uso en sistemas de doble propósito a pastoreo bajo un amplio rango de condiciones ambientales (Pearson, 2000) no hay evidencia de una diferencia de importancia económica en producción entre animales de $1 / 2$ y $5 / 8$ herencia europea. Existe insuficiente mano de obra por hectáreas en los hatos, por lo que no permiten realizar eficientemente las actividades pecuarias, siendo en 0,08 obreros/ha, en la mayoría de los casos las tareas lo hace el mismo propietario y eventualmente algún miembro de la familia, sin embargo actualmente la mano de obra familiar es muy restringido debido a que los jóvenes están migrando a las ciudades para realizar estudios superiores así como en busca de oportunidades laborales, los que están en edad escolar de igual manera.

Existe una tradición ganadera de muchos años en este sector como lo muestra la edad de los productores, sin embargo, se ha descuidado esta actividad evidenciado en la baja producción de leche por lo que los ganaderos deben conocer estos datos como resultado de este proceso de caracterización para replantear su situación productiva.

\section{Características cualitativas de los hatos productores de leche}

- Manejo: Según la tabla 2, se tienen los siguientes resultados respecto a las características cualitativas. 
Tabla 2. Frecuencia y porcentaje de variables cualitativas de hatos ganaderos

\begin{tabular}{|c|c|c|}
\hline VARIABLES & FRECUENCIA & PORCENTAJE \% \\
\hline \multicolumn{3}{|l|}{ Manejo } \\
\hline \multicolumn{3}{|l|}{ - Época de partos } \\
\hline Mayor precipitación (noviembre-abril) & 8 & 30,8 \\
\hline $\begin{array}{l}\text { Menor precipitación (mayo- octubre). } \\
\text { Numero de ordeños }\end{array}$ & 18 & 69,2 \\
\hline Un ordeño & 25 & 96,2 \\
\hline Dos ordeños & 1 & 3,8 \\
\hline \multicolumn{3}{|l|}{ - Registros de producción } \\
\hline $\mathrm{Si}$ & 17 & 65,4 \\
\hline No & 9 & 34,6 \\
\hline \multicolumn{3}{|l|}{ - Control de calidad de la leche } \\
\hline $\mathrm{Si}$ & 0 & 0 \\
\hline No & 26 & 100 \\
\hline \multicolumn{3}{|l|}{ - Equipos e instalaciones } \\
\hline No cuenta con sala de ordeño & 12 & 46,2 \\
\hline Sala de ordeños & 14 & 53,8 \\
\hline Equipo de ordeño & 0 & 0 \\
\hline \multicolumn{3}{|l|}{ Social - educativo } \\
\hline \multicolumn{3}{|l|}{ - Dedicación del productor } \\
\hline Exclusiva & 5 & 19,2 \\
\hline Parcial + otras actividades agropecuarias & 13 & 50,0 \\
\hline Parcial + actividades no agropecuarias & 8 & 30,8 \\
\hline \multicolumn{3}{|l|}{ - Nivel de estudio del productor } \\
\hline Sin estudios & 0 & 0 \\
\hline Primaria & 11 & 42,3 \\
\hline Secundaria, & 3 & 11,5 \\
\hline Técnica superior & 2 & 7,7 \\
\hline Universidad & 10 & 38,5 \\
\hline \multicolumn{3}{|l|}{ - Grado de instrucción del obrero } \\
\hline Sin estudios & 5 & 19,2 \\
\hline Primaria & 17 & 65,4 \\
\hline Secundaria & 3 & 11,5 \\
\hline Técnico productivo & 1 & 3,8 \\
\hline \multicolumn{3}{|l|}{ Alimentación } \\
\hline \multicolumn{3}{|l|}{ - Pasturas introducidas } \\
\hline B. brizantha, & 21 & 80,8 \\
\hline B. decumbens & 4 & 15,4 \\
\hline B. humidícula & 1 & 3,8 \\
\hline \multicolumn{3}{|l|}{ - Suplementación mineral } \\
\hline $\mathrm{Si}$ & 26 & 100 \\
\hline No & 0 & 0 \\
\hline
\end{tabular}


La mayoría de los productores ganaderos no hace una planificación de pariciones, sin embargo el mayor número de nacimientos ocurrió en los meses de menor precipitación pluvial que se da de mayo a octubre con un porcentaje de 69,2\%, los meses de julio y agosto son más críticos las sequías, por lo que los terneros que nazcan en este periodo tienen problemas de alimentación por escasez de forrajes al igual que las vacas tienen problemas de alimentación por consiguiente menor producción de leche tanto para la lactación como para la venta. El mes y/o época de nacimiento, afirma, Ossa et al. (2005), que están estrechamente relacionados con las variaciones climáticas y la disponibilidad de alimentos, en el invierno la oferta de forraje y la calidad de las pasturas aumentan.

Los que realizan un solo ordeño es el $96,2 \%$ de los productores en horas de la mañana a partir de las 05:00 a.m., en algunos casos para esperar al acopiador o la venta directa en el predio o en la ciudad, sin ningún tratamiento de refrigeración o químico (peróxido de hidrógeno por ejemplo) hecho que origina una baja de calidad de la leche. El 3,8\% realiza dos ordeños, el primero a partir de las 04:00 a.m. y el segundo a partir de las 02:00 p.m., en este caso la cantidad de leche de ambos ordeños se destina a la producción de queso, es el caso de la Granja Ganadera Km 17 - UNAP. El 65,4 \% de los productores llevan un registro de producción y el 34,6\% no lo hace, debido a que desconocen la importancia ya que esta información permite predecir oportunamente los posibles problemas, si no se lleva registro demuestra que los ganaderos no están manejando adecuadamente sus hatos, Vera y Riera (2004).

El 100\% de los productores no realizan control de calidad de la leche, probablemente se debe que no existe diferenciación en el precio; Cortés et al. (2012) afirma que los productores de los llanos orientales de Colombia argumentan que el eslabón industrial no les reconocía pagos diferenciales por la calidad de leche que entregaban a la planta. Las salas de ordeño es parte de la infraestructura muy importante, básicamente por el confort de las vacas y la higiene con la que debe desarrollarse esta labor, el 12\% no cuenta con estas instalaciones y el $14 \%$ si lo tiene, pero con muchas deficiencias, no cuentan colector de aguas servidas y otros desechos orgánicos, así como el equipo para el tratamiento de estas.

Social - Educativo: En cuanto a la dedicación de los propietarios que tienen sobre los hatos ganaderos, el 50\% hace otras actividades agropecuarias, refieren que sobre las actuales áreas de pastizales, tienen previsto cambiar por cultivos de cacao, palma aceitera o plátano, debido a que se está ofertando mejores condiciones técnicas de producción y comercialización a través de USAID, y el 30,8\% tienen otras actividades, relacionadas al sector público y el comercio, cuyas tareas está a cargo de un obrero, esto está conllevando a la disminución de la producción de leche inclusive a disminuir 
el número productores, Herrera (2009), afirma que existen pequeños productores sin infraestructura que tienen una alta dependencia de esta actividad.

Se destaca que el 42,3\% de los propietarios tienen educación primaria, Smith et al. (2002) indica que el nivel educacional es un factor limitante para la incorporación de tecnologías que permitan alcanzar mayores niveles productivos y de eficiencia. El $38,5 \%$ tienen estudios superiores universitarios sin embargo se dedican a otras actividades, generalmente trabajan en el sector público por lo que no garantiza la eficiencia en la producción de la leche. Los obreros en su mayoría tienen educación primaria $(65,4 \%)$ y sin ningún estudio $(19,2 \%)$, lo que se atribuye que es necesaria una capacitación y permanente monitoreo.

Alimentación: La especie forrajera de mayor importancia actualmente es la Brizantha (Brachiaria brizantha) en un 80,8 \%, probablemente, se deba a que el Fondo Nacional de Ganadería Lechera - Loreto (FONGAL), promocionó entre los productores, esta especie forrajera, como promisoria para producción de leche, los que hicieron un manejo adecuado lo recomiendan, refieren tener buenos resultados, pero en muchos predios está siendo absorbido por otras especies nativas.

Uno de los puntos críticos, es el control de calidad de la leche que conlleva a pérdidas que está relacionado con el bajo nivel educativo de la mayoría de los productores sumado a otros factores de la producción. Asimismo, el Estado promociona otras de actividades agrícolas, debido a políticas gubernamentales tanto a nivel local y regional, constituyéndose una amenaza en la continuidad de la producción ganadera en esta cuenca.

Tipificación de los hatos ganaderos productores de leche de la cuenca baja del río Shanusi

Se utilizó el método de K-medias porque permite procesar un número ilimitado de casos, partiendo de los casos individuales intentan ir agrupando hasta llegar la formación grupos o conglomerado homogéneos. En la tabla 3 se muestra la formación de conglomerados.

\section{Características de los 3 conglomerados encontrados según el Método K-media}

Conglomerado 1, lo conforman el 46,15\% de casos; crianza de sistema extensivo con mediana producción de leche, se caracteriza por el mayor número de vacas en producción, con mayor cantidad de cebuinos.

Conglomerado 2, lo conforman el 42,30 \%; crianza de sistema extensivo, con menor producción de leche, tienen el menor número de vacas en producción, con mayor número de vacas europeas. 
Conglomerados 3, lo conforman el 11,53\%, crianza extensiva, con mayor producción de leche, con regular número de vacas en producción con respecto a los conglomerados anteriores y con regular cantidad de cebuinos.

Tabla 3. Análisis de conglomerado de K-medias de las variables cuantitativas

\begin{tabular}{lccc}
\hline VARIABLES & 1 & 2 & 3 \\
\hline Edad del productor & 52,00 & 50,91 & 47,00 \\
Producción de leche & 6,50 & 5,73 & 19,00 \\
Precio de leche & 0,85 & 0,85 & 0,90 \\
Vaca en producción & 7,25 & 3,09 & 5,00 \\
Pasturas mejoradas & 13,42 & 10,00 & 10,00 \\
Carga animal & 1,89 & 1,81 & 1,08 \\
Número de obreros & 0,07 & 0,09 & 0,08 \\
Total vacunos & 37,00 & 35,00 & 36,00 \\
Vacuno europeos & 26,00 & 63,00 & 40,00 \\
Vacuno cebuíno & 69,00 & 37,00 & 60,00 \\
Fertilizantes & 0,00 & 0,00 & 120,00 \\
\hline Porcentaje de casos en los conglomerados & 46,15 & 42,30 & 11,53 \\
\hline
\end{tabular}

\section{CONCLUSIONES}

Los hatos ganaderos se caracterizan por poca producción de leche con bajo precio, reducido número de vacas en ordeño, con especies forrajeras introducidas al pastoreo continuo con escasa fertilización, carga animal que no guarda relación con la producción de forraje.

Los productores se dedican a otras actividades agrícolas debido a las oportunidades de mercado, apoyo técnico y económico que reciben de organizaciones públicas y privadas desplazando a la actividad lechera. El nivel educativo de la mayoría de propietarios dificulta la adopción de nuevas tecnologías.

Los hatos se tipifican en tres conglomerados, el Conglomerado 1 de mediana producción, Conglomerado 2 menor producción y Conglomerado 3 con mayor producción de leche, respectivamente. Además, se caracterizan por ser sistemas extensivos. 


\section{RECOMENDACIONES}

Los ganaderos lecheros de la cuenca baja del río Shanusi deben buscar el fortalecimiento de la asociación de productores para facilitar el acercamiento a las instituciones públicas y privadas que promocionan y forman parte de la cadena productiva para gestionar: capacitación, mejora genética, infraestructura y canales de comercialización.

\section{Agradecimiento}

A los productores ganaderos de la cuenca baja del rio Shanusi que han contribuido con la realización de este trabajo de investigación y al Ing. Luis Arévalo López Gerente Regional IIAP San Martín.

\section{REFERENCIAS BIBLIOGRÁFICAS}

Andrade, Roy et al. 2008. «Características productivas y de gestión de fincas lecheras en Boyacá». Revista MVZ. Vol. 13, N 2. Córdoba Universidad de Córdoba Montería.1333 - 1342

Avilez, Juan et al. 2010. «Caracterización productiva de explotaciones lecheras empleando metodología de análisis multivariado». Revista Científica, FCV-LUZ. Vol. XX, $\mathrm{N}^{\circ}$ 1, Universidad de Zulia. $74-80$.

Cortés, Jimmy et al. 2012. «Características estructurales del sistema de producción con bovinos doble propósito en el trópico húmedo colombiano». Revista Colombiana de Ciencias Pecuarias. Vol. 25. N². Universidad Nacional de Colombia. 229 - 239.

Herrera, Nelsy. 2009. «Identificación de la capacidad empresarial y la eficiencia de los productores de leche de Guamal». Departamento del Meta Pontificia Universidad Javeriana. Tesis de Maestría en Desarrollo Rural. 101.

Ossa, Gustavo et al. 2005. «Efectos del medio y la herencia sobre el peso al destete de terneros de la raza romosinuano». Programa Nacional de Recursos Genéticos y Biotecnología Animal, CORPOICA. C.I. Turipaná. Montería. MVZ-Córdoba. Vol. 10. N 2. 673-683.

Pearson, Lucia. 2000. Cruzamiento para la producción de leche en América Tropical: evidencia de Venezuela. III Simpósio Nacional de Melhoramiento Animal. Universidad Central de Venezuela Instituto de Producción Animal, Facultad de Agronomía y Ciencias Veterinarias. Maracay - Venezuela. 38-46.

Requelme, Narcisa y Bonifaz, Nancy. 2012. «Caracterización de sistemas de producción lechera de Ecuador». Centro de Investigación de la Leche CILEC. Vol. 15. $\mathrm{N}^{\circ} 1$ Universidad Politécnica Salesiana del Ecuador. 55-69.

Ríos, Jorge. 2007. Enfoques integrales de producción ganadera en la amazonia peruana. XX Reunión ALPA, XXX Reunión APPA-Cusco-Perú. 234-240. 
Ruiz, Heidy. 2011. Estudio sobre la posición competitiva del sector lácteos del distrito de Soritor - Alto Mayo región San Martin. Universidad Nacional de San Martín. Facultad de Ingeniería Agroindustrial, Tesis. 134.

Salamanca, Arcenio y Bentez, Jannet. 2012. «Producción de leche de vacas mestizas del Sistema Doble Propósito en el municipio de Arauca». Revista electrónica de Veterinaria. Vol. 13. No 7. Málaga. 1-15.

Smith, Ricardo et al. 2002. «Caracterización de sistemas productivos lecheros en la X región de Chile mediante análisis multivariable». Revista Agricultura Técnica. Vol. 62. $\mathrm{N}^{\circ} 3.375-395$.

Vásquez, Ludwing. 2007. Caracterización de cinco fundos ganaderos en la zona de la carretera Yurimaguas-Munichis. Universidad Nacional de la Amazonía Peruana. Facultad de Zootecnia. <http://repositorio.unapiquitos.edu.pe/handle/UNAP/1749> [Consulta: 09-10-2017]

Vera, Antonio y Riera, Luis. 2004. Desarrollo de alternativas silvopastoriles para rehabilitar pastizales en zona norte de la región Amazónica ecuatoriana. El Coca, EC. Estación Experimental Napo-Payamino. INIAP. Manual 59.

Recepción: 03/09/2018

Aceptación: 12/10/2018

\section{Correspondencia}

Marco Mathios Flores

mathiosflores@hotmail.com 